\title{
Detection of Autoantibodies to Annexin A11 in Different Types of Human Cancer
}

\author{
Jin Song • Xiaer Sun • Lori J. Sokoll • Masatoshi Maki • \\ Yuan Tian • Daniel W. Chan $\cdot$ Zhen Zhang
}

Published online: 9 May 2009

(C) Humana Press 2009

\begin{abstract}
Introduction Annexin A11 was previously identified as an autoantigen in $4.1-10.1 \%$ of patients with various systemic autoimmune diseases. In this study, an enzyme-linked immunosorbent assay (ELISA) was developed to investigate the occurrence and features of anti-annexin A11 autoantibodies in sera from patients with different types of cancer. Methods The recombinant protein of GST fused to the Nterminal domain (1-175 residues) of human annexin A11 was expressed and used as antigen in ELISA. A total of 246 serum specimens were analyzed, which includes sera from healthy women (77), patients with ovarian cancer (72), breast cancer (18), colon cancer (19), pancreatic cancer (20), prostate cancer (20), and diabetes (20).

Results The overall titer of anti-annexin A11 autoantibodies in ovarian cancer patients (or primary tumors only) was found much higher than that in healthy controls $(P<0.05)$. At the cut-off value designating positive reaction, antiannexin A11 autoantibodies were detected in 12.5\% (5/40) of primary ovarian cancer patients with a significant

J. Song $\cdot$ X. Sun $\cdot$ L. J. Sokoll $\cdot$ Y. Tian $\cdot$ D. W. Chan $\cdot$ Z. Zhang

Center for Biomarker Discovery, Department of Pathology,

Johns Hopkins Medical Institutions,

Baltimore, MD, USA

M. Maki

Laboratory of Molecular and Cellular Regulation,

Department of Applied Molecular Biosciences,

Graduate School of Bioagricultural Sciences, Nagoya University,

Furo-cho, Chikusa-ku,

Nagoya, Japan

\section{Z. Zhang $(\bowtie)$}

Department of Pathology, Johns Hopkins Medical Institutions,

1550 Orleans Street,

Baltimore, MD 21231, USA

e-mail: zzhang7@jhmi.edu
\end{abstract}

difference from $2.6 \%(2 / 77)$ of the healthy controls $(P<$ $0.05)$, but only in $6.25 \%(2 / 32)$ of recurrent tumors. ROC curve demonstrated the potential diagnostic value of antiannexin A11 autoantibodies in primary ovarian cancer patients with an AUC of 0.62 (0.52-0.73). Anti-annexin A11 autoantibodies were also detected in 5.26\% (1/19) of colon cancer and 10\% (2/20) of diabetes patients but without significant difference from the healthy controls.

Conclusion A convenient assay to detect anti-annexin A11 autoantibodies in patients was developed, and the experimental data are promising but need to be expanded to address their biological/clinical relevance.

Keywords Autoantibody - Annexin A11 - Cancer .

Enzyme-linked immunosorbent assay

$\begin{array}{ll}\text { Abbreviations } & \\ \text { ELISA } & \text { Enzyme-linked immunosorbent assay } \\ \text { GST } & \text { Glutathione } S \text {-transferase } \\ \text { ANXA11N } & \text { N-terminal regulatory domain of annexin } \\ & \text { A11 } \\ \text { SDS-PAGE } & \text { Sodium dodecyl sulfate-polyacrylamide } \\ & \text { gel electrophoresis }\end{array}$

\section{Introduction}

There is increasing evidence for an immune response to human cancer, demonstrated in part by the identification of autoantibodies against some intracellular and surface antigens in patients with different types of cancer [1-3]. The identification of these tumor-associated antigens/autoantibodies may have utility in cancer screening, diagnosis, predicting prognosis, and immunotherapy. Annexin A11 is a member of the 
annexin superfamily of $\mathrm{Ca}^{++}$signal transduction proteins associated with cell growth and differentiation [4-6]. All of the annexin members share a conserved C-terminal domain containing four or eight repeats of around 70 amino acids that is presumed to be involved in phospholipid binding in a $\mathrm{Ca}^{++}$dependent way. Each member contains a unique N-terminal domain that strongly varies both in length and in sequence $[7,8]$. Previously, annexin A11 was identified as an autoantigen in a substantial number of sera (4.1-10.1\%) from patients with various systemic autoimmune diseases, including rheumatoid arthritis, systemic lupus erythematosus, Sjögren's syndrome, systemic sclerosis, and Raynaud's phenomenon, but was rarely detected in sera from healthy controls and in patients with infectious diseases [9-12]. The majority of these anti-annexin A11 autoantibodies belong to the IgG class, consistent with an antigen-driven mechanism of autoantibody production, in contrast to autoantibodies to other annexins that are primarily of the IgM isotype. Antiannexin A11 autoantibodies do not cross-react with other annexin members, corroborating that they recognize the unique N-terminal domain of annexin A11 [10]. Most recently, in a genome-wide association study, annexin A11 was identified as a new susceptibility locus for sarcoidosis [13]. How annexin A11 participates in the pathogenesis of human cancers and whether a similar mechanism in autoimmune diseases might be involved in human immune responses in cancer remains to be established.

Recently, we showed that annexin A11 is associated with cisplatin resistance and related to tumor recurrence in ovarian cancer patients [14]. Using immunohistochemical staining, the expression level of annexin A11 in first recurrence, postchemotherapy ovarian cancers was found to be much lower than that in primary, pre-chemotherapy ovarian cancers [14]. In this study, an enzyme-linked immunosorbent assay (ELISA) was developed to investigate the occurrence and features of autoantibodies against annexin A11 in sera from patients with ovarian cancer and from healthy controls. For comparison, sera from patients with other types of human cancer as well as diabetes were also analyzed.

\section{Materials and Methods}

\section{Patients and Controls}

In this study, a total of 246 serum specimens archived at the Johns Hopkins Hospital were analyzed, which includes sera from 77 healthy women [mean (SD) age, 48 (12) years]; 72 patients with stage III/IV (45 and 27 cases, respectively) ovarian cancer (40 primary and 32 recurrent tumors) [58 (14) years]; 18 patients with stage 0 , I, II, or III (three, five, seven, and three cases, respectively) breast cancer [53 (16) years]; 19 patients with stage I, II, or III (one, ten, and eight cases, respectively) colon cancer [69 (16) years]; and groups of 20 patients each with stage II or III (four and 16 cases, respectively) pancreatic cancer [66 (8) years], stage I, II, or III (one, 12, and seven cases, respectively) prostate cancer [58 (8) years], or diabetes [52 (18) years]. All patients with breast, colon, or pancreatic cancer and diabetes were female. All serum samples had been processed promptly after collection and stored at $2-8^{\circ} \mathrm{C}$ for a maximum of $48 \mathrm{~h}$ before freezing at $-70^{\circ} \mathrm{C}$. Besides the sera from the patients with recurrent ovarian cancers, who underwent primary debulking surgery followed by routine combined chemotherapy, all other sera were obtained before treatment and surgery. This study was approved by the Institutional Review Board of Johns Hopkins Medical Institutions.

\section{Expression and Purification of Recombinant Proteins}

A cDNA fragment encoding the N-terminal domain of human annexin A11 (ANXA11N, amino acid residues 1175) was obtained by cutting the full-length cDNA clone pGADGH-annexin A11 with $B g / \mathrm{II}$ and inserting it into the BamHI site of glutathione $S$-transferase (GST) fusion vector pGEX-4T-2 followed by insertion of a synthetic oligonucleotide block at the NotI site to add a hexahistidine $(\times$ His) tag at its C-terminus. E. coli BL21 cells were transformed with the resultant plasmid (pGST-ANXA11N) [15]. The fusion protein of GST-ANXA11N was expressed in E. coli BL21 cells and purified by affinity chromatography using glutathione-Sepharose 4B (Amersham Biosciences) according to the manufacturer's instructions. GST-ANXA11N was eluted from the Sepharose beads with $10 \mathrm{mM}$ reduced glutathione in $50 \mathrm{mM}$ Tris- $\mathrm{HCl}(\mathrm{pH} 8.5)$ and stored at $4^{\circ} \mathrm{C}$ until use. The purity of the recombinant protein was examined by sodium dodecyl sulfate-polyacrylamide gel electrophoresis (SDSPAGE) and immunoblot analyses with rabbit anti-GST (Novus Biologicals) and rabbit anti-annexin A11 (ProteinTech Group) polyclonal antibodies.

Enzyme-linked Immunosorbent Assay

Purified recombinant protein was diluted in the coating buffer [ $1 \times$ phosphate-buffered saline (PBS), $\mathrm{pH} 7.4]$ to a final concentration of $12 \mu \mathrm{g} / \mathrm{ml}$ and dispensed to 96-well plates (MaxiSorp plate, Nalge Nunc International) at $50 \mu 1 /$ well. The plates were incubated overnight at $4{ }^{\circ} \mathrm{C}$. The plates were blocked with the blocking buffer $(5 \% \mathrm{w} / v$ milk in $1 \times \mathrm{PBS}$, $\mathrm{pH} 7.4)$ for $1 \mathrm{~h}$ at $37^{\circ} \mathrm{C}(100 \mu \mathrm{l} /$ well $)$, then washed three times with the washing buffer ( $1 \times \mathrm{PBS}, \mathrm{pH} 7.4$ with $0.05 \%$ $v / v$ Tween 20). Human sera were diluted 1:100 in the blocking buffer and applied to the plates (100 $\mu \mathrm{l} / \mathrm{well})$, and the plates were then incubated for $1 \mathrm{~h}$ at $37^{\circ} \mathrm{C}$, washed three times with the washing buffer. Horseradish peroxidase- 
conjugated goat anti-human $\operatorname{IgG}(\mathrm{H}+\mathrm{L})$ antibody (Promega Corporation) was diluted 1:10,000 in the blocking buffer and applied to the plate $(100 \mu \mathrm{l} /$ well $)$, and the plates were then incubated for $1 \mathrm{~h}$ at $37^{\circ} \mathrm{C}$. After washing three times with the washing buffer, the plates were incubated with the substrate solution [tetramethylbenzidine (TMB); KPL Inc.] at $100 \mu \mathrm{l} /$ well for $20 \mathrm{~min}$, and the reaction was stopped with the equal volume of stop solution $\left(0.18 \mathrm{M} \mathrm{H}_{2} \mathrm{SO}_{4}\right)$. The absorbance was measured at $450 \mathrm{~nm}$. Each serum was assayed in triplicate for the reactivity with GST-ANA11N. Sera from two individuals with a high titer were pooled as a standard, with serial dilutions forming the standard curve. The cut-off value was determined from the mean OD of 77 healthy control sera $+2 \mathrm{SD}$.

\section{SDS-PAGE and Immunoblot Analysis}

Purified GST-ANXA11N recombinant proteins (200 ng) were electrophoresed on a 4-15\% gradient SDS-PAGE and electroblotted on nitrocellulose membranes (Bio-Rad). After blocking with $1 \times$ PBS containing $0.05 \%$ Tween-20 and $3 \%$ non-fat milk for $30 \mathrm{~min}$ at room temperature, the nitrocellulose strips were incubated for $1 \mathrm{~h}$ at room temperature with a 1:200 dilution of serum. The bound autoantibodies were visualized with horseradish peroxidase-conjugated goat anti-human $\operatorname{IgG}(\mathrm{H}+\mathrm{L})$ antibody (1:2,500 dilution; Promega Corporation) and enhanced chemiluminescence (Amersham Biosciences). The rabbit anti-GST and rabbit anti-annexin A11 polyclonal antibodies were diluted at 1:1,000 and 1:600, respectively.

Absorption of Autoantibodies with Recombinant GST-ANXA11N

Sera $(2 \mu \mathrm{l})$ were diluted in $800 \mu \mathrm{l}$ of the blocking buffer, and incubated for $2 \mathrm{~h}$ at room temperature with $15 \mu \mathrm{g}$ of GSTANXA11N recombinant protein. This mixture was centrifuged at $10,000 \times \mathrm{g}$ for $10 \mathrm{~min}$, and the supernatant was then used for the analysis by ELISA with GST-ANXA11N recombinant protein as the coating antigen as described above. Each serum was assayed in triplicate for the comparison of autoantibody titers of the pre- and post-incubations.

\section{CA125 Measurement}

Serum CA125 levels were measured with a two-site immunoenzymometric assay on the TOSOH AIA-600 II analyzer (Tosoh Bioscience), according to the manufacturer's protocol.

\section{Statistical Analyses}

Statistical significance of results was assessed using the unpaired $t$ test and the Pearson $\chi^{2}$ test. Differences with
$P<0.05$ were considered statistically significant. ROC curve analysis was performed to evaluate the diagnostic potential of autoantibodies to annexin A11 in primary ovarian cancer patients. All of the statistical analyses were performed using Statistica 6.1 (Statsoft) and Analyze-it 1.71 (Analyze-it Software).

\section{Results}

In this study, the recombinant protein of GST fused to the N-terminal domain (1-175 amino acid residues) of human annexin A11 was expressed and used as antigen in ELISA. The purity of the recombinant protein was verified by SDSPAGE and immunoblot analyses with rabbit anti-GST and rabbit anti-annexin A11 polyclonal antibodies (data not shown). A total of 246 serum specimens were tested for the presence of autoantibdies against annexin A11, which includes sera from healthy women (77), patients with ovarian cancer (72), breast cancer (18), colon cancer (19), pancreatic cancer (20), prostate cancer (20), and diabetes (20). A serum pool, which was mixed from two ovarian cancer patient sera with high anti-annexin A11 autoantibody titer, was serially diluted with the blocking buffer and analyzed by ELISA. As shown in Fig. 1, the dynamic measurement range of anti-annexin A11 autoantibody titers spans a more than 16-fold difference. The ranges of antiannexin A11 autoantibody titers in different types of human cancer and diabetes as well as healthy controls obtained from ELISA are shown in Fig. 2. The experimental results showed that the overall titer of autoantibodies to annexin A11 in ovarian cancer patients was found to be much higher than that in healthy controls $(P=0.024)$. More specifically, primary ovarian cancer patients showed an increased anti-annexin A11 autoantibody titer compared to healthy controls $(P=0.017)$. Although the overall titer of autoantibodies to annexin A11 in recurrent ovarian cancer patients was also found to be increased compared with that of healthy controls or decreased compared with that of primary ovarian cancer patients, a statistically significant difference was not reached. There were no significant differences in anti-annexin A11 autoantibody titers between other diseases, including breast, colon, pancreatic, and prostate cancers and diabetes, and healthy controls. At the cut-off value (mean $\mathrm{OD}+2 \mathrm{SD}$ of 77 healthy controls), autoantibodies to annexin A11 were detected in $12.5 \%$ (5/ 40) of primary ovarian cancer patients with a significant difference from $2.6 \%(2 / 77)$ in healthy controls $(P=0.032)$. Autoantibodies were detected in only $6.25 \%(2 / 32)$ of patients with recurrent tumors which was not significantly different from healthy controls (Table 1). Autoantibodies to annexin A11 were also detected in 5.26\% (1/19) of colon cancer and $10 \%(2 / 20)$ of diabetes patients but the 


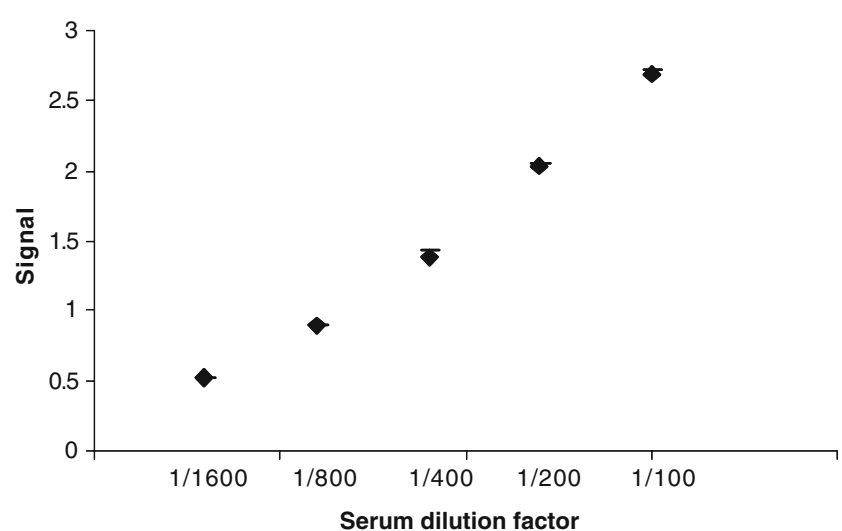

Fig. 1 Dynamic measurement range of anti-annexin A11 autoantibody titers in serum. A serum pool with high anti-annexin A11 autoantibody titer was serially diluted with the blocking buffer and analyzed by ELISA. As shown, the dynamic measurement range of anti-annexin A11 autoantibody titers spans a more than 16-fold difference. $X$-axis, serum dilution factor; $Y$-axis, signal in OD value

difference from healthy controls was not statistically significant. ROC curve analysis demonstrated the potential diagnostic value of autoantibodies to annexin A11 in primary ovarian cancer patients with an AUC of 0.62 (0.52-0.73, $P=0.011$ ) (Fig. 3).

To validate the ELISA results, all of the ELISA positive sera were examined by SDS-PAGE and immunoblot analysis. The sera that were positive in ELISA were also found to be strongly positive in immunoblot analysis, whereas the representative ELISA negative sera did not have positive reactions in immunoblotting (Fig. 4). The positive sera mainly recognized the annexin A11 related bands but not GST only bands, which suggested the specificity of the autoantibodies. The specificity of GSTANXA11N as an antigen for ELISA was also confirmed by the absorption studies (Fig. 5). All of the ELISA positive sera were pre-incubated with the recombinant protein and used subsequently for the ELISA. One negative healthy women serum was also tested as control. Reactivity of the positive sera decreased substantially after pre-incubation with GST-ANXA11N, but not in the control serum. There was a significant difference between the autoantibody titers of pre- and post-incubations $(P<0.001)$.

Of the 72 ovarian cancer patients, there were 47 cases with available CA125 measurements (range $=18.02$ to $21,421 \mathrm{U} / \mathrm{ml} ;$ mean $\pm \mathrm{SD}=2,586.791 \pm 5,301.855 \mathrm{U} / \mathrm{ml})$. No significant correlation between the annexin A11 autoantibody titer and CA125 measurement was observed $(r=0.204$, data not shown).

\section{Discussion}

Circulating autoantibodies against tumor-associated antigens have been observed in patients with different types of cancer and may serve as biomarkers for the screening, diagnosis, prognosis, or monitoring of cancer [1-3]. For example, the autoantibody to p53 is detectable in serum and may predict subsequent development of cancer [3, 16]. However, so far the clinical utility of tumor-associated autoantibodies has been hampered by the low frequencies to each particular antigen and the heterogeneity of antibodies in cancer patients. It is not clear why only a subset

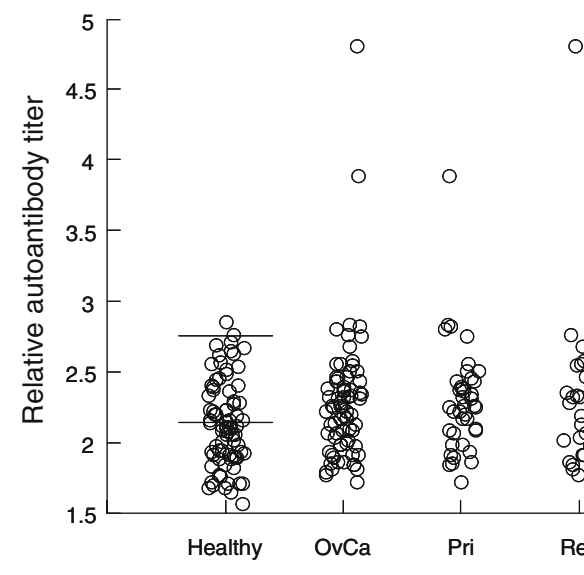

Fig. 2 Scatter plot of anti-annexin A11 autoantibody titers in different types of human cancer. The ranges of anti-annexin A11 autoantibody titers in different types of human cancer and diabetes as well as healthy controls obtained from ELISA were shown here. The $Y$-axis represents the relative anti-annexin A11 autoantibody titers. The $X$ axis represents the type of serum samples: Healthy, healthy women; $\mathrm{OvCa}$, ovarian cancer (Pri, primary tumor; Rec, recurrent tumor);

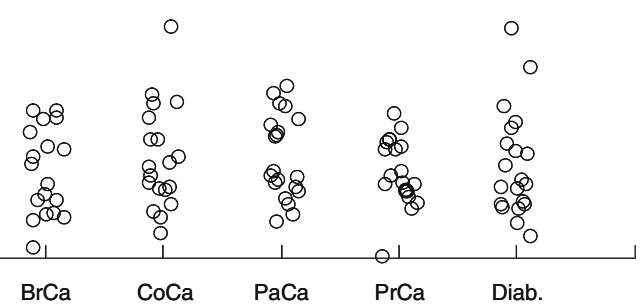

$\mathrm{BrCa}$, breast cancer; $\mathrm{CoCa}$, colon cancer; $\mathrm{PaCa}$, pancreatic cancer; $\operatorname{PrCa}$, prostate cancer; Diab, diabetes. The mean and 2 SD of 77 healthy women sera were indicated by horizontal lines. The overall titer of anti-annexin A11 autoantibodies in ovarian cancer patients (or primary tumors only) was found much higher than that in healthy controls $(P<0.05)$ 
Table 1 Frequency of autoantibodies to annexin A11 in different types of human cancer

\begin{tabular}{lll}
\hline Cancer & No. of patients & Positive $(\%)^{\mathrm{a}}$ \\
\hline Ovarian & 72 & $7(9.72 \%)$ \\
Primary & 40 & $5(12.5 \%)^{*}$ \\
Recurrent & 32 & $2(6.25 \%)$ \\
Breast & 18 & $0(0)$ \\
Colon & 19 & $1(5.26 \%)$ \\
Pancreas & 20 & $0(0)$ \\
Prostate & 20 & $0(0)$ \\
Diabetes & 20 & $2(10 \%)$ \\
Healthy & 77 & $2(2.6 \%)$ \\
\hline
\end{tabular}

The cut-off value designating positive reaction was the mean OD of 77 healthy women sera $+2 \mathrm{SD}$

${ }^{\text {a }} P$ value relative to healthy controls: ${ }^{*} P<0.05$

of patients with a tumor type develop a humoral response to a particular antigen. Studies of these autoantigens/autoantibodies could be used to understand how cellular proteins become immunogenic and drive an immune response in cancer. Such tumor-associated antigens may also have utility in immunotherapy against the disease.

The occurrence of autoantibodies against different annexin members has been reported before [4, 9-12, 17]. Annexin A11 was initially identified as an autoantigen in $4.1-10.1 \%$ of patients with various systemic autoimmune diseases, but not in healthy controls [9-12]. Although autoantibodies to other annexins seem to be primarily of the IgM isotype, anti-annexin A11 reactivity is mostly of $\operatorname{IgG}$ isotype, demonstrating that annexin A11 is a distinct autoantigen compared to other members [10]. These observations suggested that anti-annexin A11 autoantibodies were generated via an antigen-driven mechanism, like other well-defined disease specific autoantibodies, but not simply due to polyclonal $\mathrm{B}$ cell activation or due to chronic inflammation. Misaki et al. [10] showed that a mutant lacking a substantial region of the C-terminal part (amino acid 315-505) of the annexin A11 molecule was recognized by all anti-annexin A11 positive sera from patients with systemic autoimmune diseases as efficiently as the full-length protein. Moreover, mutants containing only (part of) the annexin A11 specific sequences (amino acid 1-191 and 1-123) showed the same behavior, strongly suggesting that the major epitope(s) recognized by all patient sera reside in the N-terminal, annexin-specific part of the protein [10]. In the present study, the recombinant protein of GST fused to the N-terminal domain (amino acid 1-175) of human annexin A11 was expressed and used as antigen in the ELISA development, and a total of 246 sera from patients with different types of cancer, diabetes, and healthy women were examined for the presence of autoantibodies to annexin A11.

The experimental results showed that the overall titer of autoantibodies to annexin A11 in ovarian cancer patients (or primary tumors only) was found to be much higher than that in healthy controls $(P<0.05)$. At the cut-off value (mean $\mathrm{OD}+2 \mathrm{SD}$ of healthy controls), autoantibodies to annexin A11 were detected in $12.5 \%$ of primary ovarian cancer patients with a significant difference from $2.6 \%$ in healthy controls $(P<0.05)$, compared to only $6.25 \%$ of patients with recurrent tumors. ROC analysis demonstrated the potential diagnostic value of autoantibodies to annexin A11 in primary ovarian cancer patients with an AUC of $0.62(0.52-0.73, P<0.05)$. Recently, we reported that annexin A11 is associated with cisplatin resistance and related to tumor recurrence in ovarian cancer patients [14]. Using immunohistochemical staining, the expression level of annexin A11 in first recurrence, post-chemotherapy ovarian cancers was found much lower than that in primary, pre-chemotherapy ovarian cancers [14]. Taken together, the anti-annexin A11 autoantibody titer in sera of ovarian cancer patients seems coincident with the annexin A11 expression level in tumor tissues. Our study suggested that anti-annexin A11 autoantibodies frequently occur in primary ovarian cancer patients in contrast to healthy controls; might appear through a similar antigen-driven mechanism in autoimmune diseases; and may be involved in the pathogenesis of ovarian cancer.

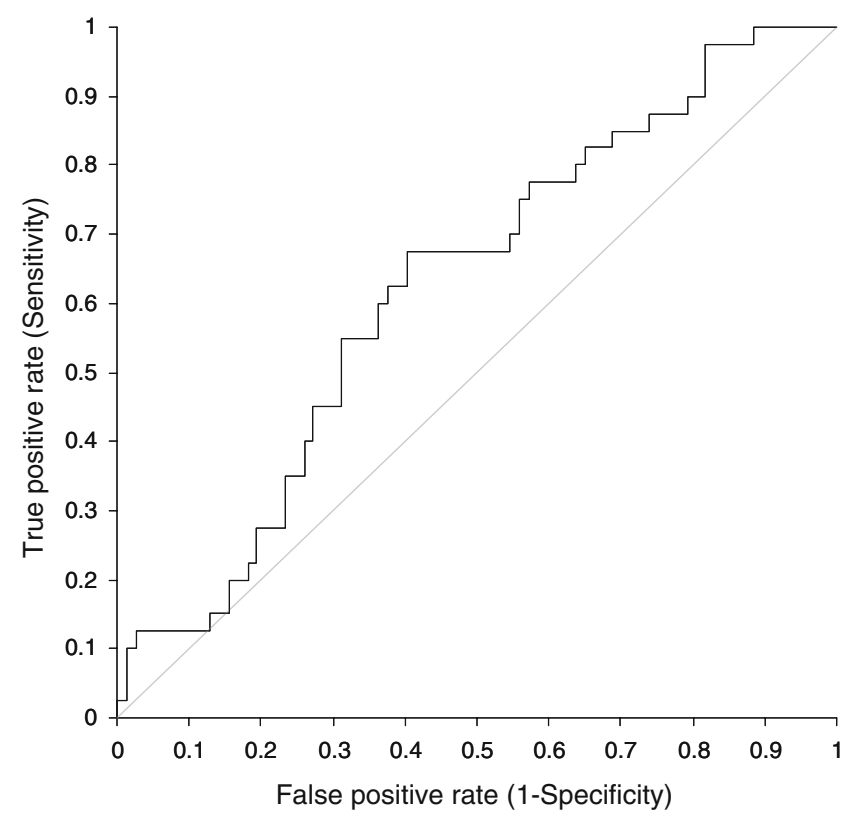

Fig. 3 ROC curve analysis. ROC curve analysis demonstrated the potential diagnostic value of autoantibodies to annexin A11 in primary ovarian cancer patients with an AUC of $0.62(0.52-0.73, P=0.011)$ 
Fig. 4 Immunoblot analysis. Representative reactivity of negative and positive sera in Western blotting against purified GST-ANXA11N recombinant protein. Lane 1, ladder; lane 2, healthy women serum, negative; lanes 3-6, four positive ovarian cancer sera; lanes $7-8$, two positive diabetes sera

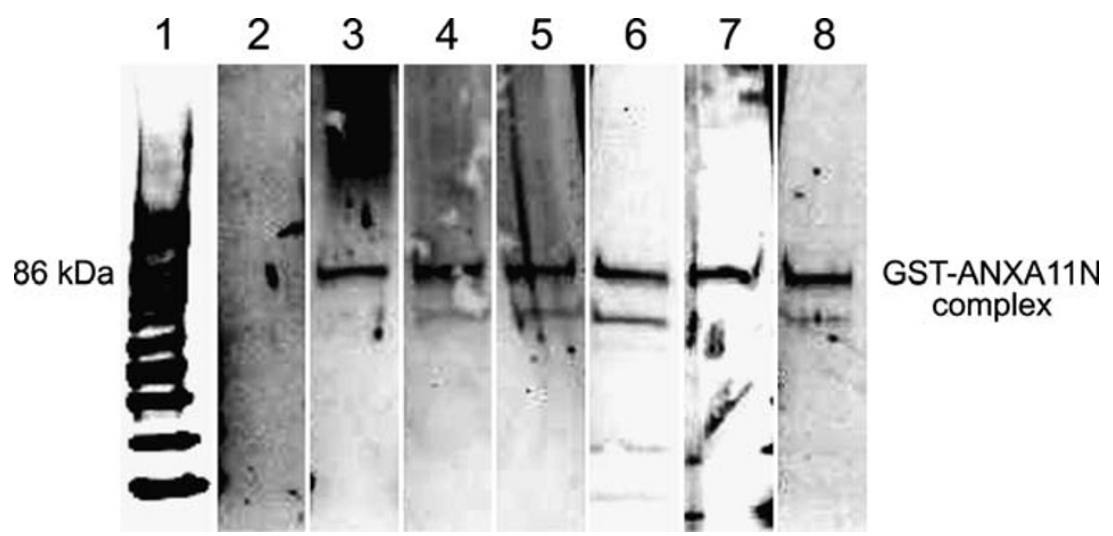

Immunogenicity may depend on the level of expression, posttranslational modification, or other types of processing of a protein, the extent of which may be variable among tumors of a similar type. Annexins are known to undergo posttranslational modification including glycosylation [18] and phosphorylation $[8,19]$. The N-terminal region of annexin A11 has been proposed to be responsible for its autoantigenicity [10], nuclear localization [20], and tyrosine phosphorylation [8]. A potential $\mathrm{N}$-linked glycosylation site is present at position 60 from the N-terminal of annexin A11 (prediction of N-glycosylation sites using NetNGlyc 1.0 Server at http://www.cbs.dtu.dk/services/NetNGlyc/). Immunoreactivity against annexin $\mathrm{A} 1$ has been found to be dependent on N-glycosylation of the protein [4]. Other factors that influence the immune response may include variability among individuals and tumors in major histocompatibility complex molecules [21, 22]. Consistent with our observations, autoantibodies against some annexins were also shown to occur quite frequently in sera from patients with malignant melanoma [23], lung cancer [4], and breast cancer [17]. It was reported that $\operatorname{IgG}$ autoantibodies reacting with the amino acid 41-74 sequence of annexin A11 were found in $11 \%$ of patients with invasive breast ductal carcinoma, whereas they were found only in $2 \%$ of non-cancer controls [17]. In a recent immunohistochemistry study, annexin A11 was found overexpressed in primary colorectal cancer and decreased in metastatic colorectal cancer [24]. In the present study, autoantibodies to annexin A11 were also detected in 5.26\% of colon cancer and $10 \%$ of diabetes patients but the difference from healthy controls was not statistically significant, possibly due to the relatively small sample size. Thus, the extent to which annexin A11 autoantibodies may occur in tumor types other than ovarian cancer requires further investigations, particularly in cancer types in which increased annexin A11 has been observed, as in the case of breast cancer [17] and colon cancer [24].

\section{Conclusion}

We present the first report of a convenient method to detect anti-annexin A11 autoantibodies in patients with different types of cancer. This study suggested that anti-annexin A11 autoantibodies frequently occur in primary ovarian cancer patients in contrast to healthy controls, and might appear through a similar antigen-driven mechanism in autoimmune diseases. These pilot study findings should be followed up in a larger case-control cohort of ovarian cancer patient sera to test the hypothesis that anti-annexin A11 autoantibodies may be involved in the pathogenesis of ovarian cancer.

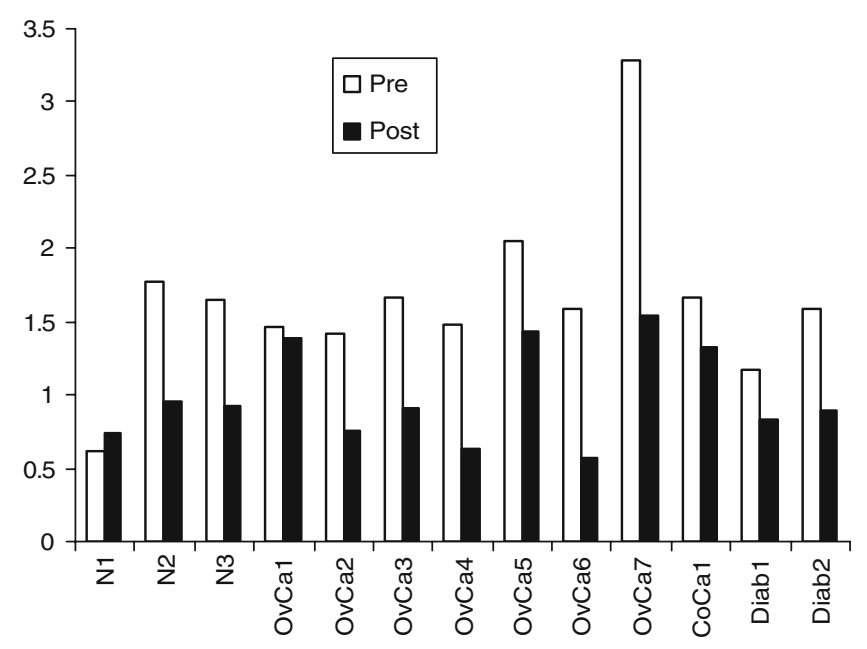

Fig. 5 Absorption of autoantibodies with recombinant GSTANXA11N. Sera were diluted in the blocking buffer, and incubated for $2 \mathrm{~h}$ at room temperature with GST-ANXA11N recombinant protein. This mixture was centrifuged at $10,000 \times g$ for $10 \mathrm{~min}$, and the supernatant was then used for the analysis by ELISA with GSTANXA11N recombinant protein as coating antigen. $N 1$, one healthy women serum, negative control; others, positive sera $(N 2, N 3$, positive healthy women sera). There is a significant difference between the autoantibody titers of pre- and post-incubations $(P<0.001)$ 
Acknowledgements This work was partially supported by funding from the National Cancer Institute Early Detection Research Network grant CA115102 (D.W.C.), National Cancer Institute grant 1P50 CA83639, and The University of Texas M. D. Anderson Cancer Center Specialized Programs of Research Excellence (SPORE) in Ovarian Cancer (Z.Z.).

\section{References}

1. Old LJ, Chen YT. New paths in human cancer serology. J Exp Med. 1998;187(8):1163-7.

2. Sahin U, Tureci O, Schmitt H, et al. Human neoplasms elicit multiple specific immune responses in the autologous host. Proc Natl Acad Sci U S A. 1995;92(25):11810-3.

3. Soussi T. p53 Antibodies in the sera of patients with various types of cancer: a review. Cancer Res. 2000;60(7):1777-88.

4. Brichory FM, Misek DE, Yim AM, et al. An immune response manifested by the common occurrence of annexins I and II autoantibodies and high circulating levels of IL-6 in lung cancer. Proc Natl Acad Sci U S A. 2001;98(17):9824-9.

5. Chetcuti A, Margan SH, Russell P, et al. Loss of annexin II heavy and light chains in prostate cancer and its precursors. Cancer Res. 2001;61(17):6331-4.

6. Monastyrskaya K, Babiychuk EB, Hostettler A, Rescher U, Draeger A. Annexins as intracellular calcium sensors. Cell Calcium. 2007;41(3):207-19.

7. Fernandez MP, Jenkins NA, Gilbert DJ, Copeland NG, Morgan RO. Sequence and chromosomal localization of mouse annexin XI. Genomics. 1996;37(3):366-74.

8. Furge LL, Chen K, Cohen S. Annexin VII and annexin XI are tyrosine phosphorylated in peroxovanadate-treated dogs and in platelet-derived growth factor-treated rat vascular smooth muscle cells. J Biol Chem. 1999;274(47):33504-9.

9. Jorgensen CS, Levantino G, Houen G, et al. Determination of autoantibodies to annexin XI in systemic autoimmune diseases. Lupus. 2000;9(7):515-20.

10. Misaki Y, Pruijn GJ, van der Kemp AW, van Venrooij WJ. The 56K autoantigen is identical to human annexin XI. J Biol Chem. 1994;269(6):4240-6.
11. Misaki Y, Van Venrooij WJ, Pruijn GJ. Prevalence and characteristics of anti-56K/annexin XI autoantibodies in systemic autoimmune diseases. J Rheumatol. 1995;22(1):97-102.

12. van Venrooij WJ, Wodzig KW, Habets WJ, de Rooij DJ, van de Putte LB. Anti-56K: a novel, frequently occurring autoantibody specificity in connective tissue disease. Clin Exp Rheumatol. 1989;7(3):277-82.

13. Hofmann S, Franke A, Fischer A, et al. Genome-wide association study identifies ANXA11 as a new susceptibility locus for sarcoidosis. Nat Genet 2008.

14. Song J, Shih Ie M, Salani R, Chan DW, Zhang Z. Annexin XI is associated with cisplatin resistance and related to tumor recurrence in ovarian cancer patients. Clin Cancer Res. 2007;13(22 Pt 1):6842-9.

15. Satoh H, Shibata H, Nakano Y, Kitaura Y, Maki M. ALG-2 interacts with the amino-terminal domain of annexin $\mathrm{XI}$ in a $\mathrm{Ca}(2+)$ dependent manner. Biochem Biophys Res Commun. 2002;291 (5):1166-72.

16. Angelopoulou K, Diamandis EP, Sutherland DJ, Kellen JA, Bunting PS. Prevalence of serum antibodies against the p53 tumor suppressor gene protein in various cancers. Int $\mathrm{J}$ Cancer. 1994;58(4):480-7.

17. Fernandez-Madrid F, Tang N, Alansari H, et al. Autoantibodies to annexin XI-A and other autoantigens in the diagnosis of breast cancer. Cancer Res. 2004;64(15):5089-96.

18. Goulet F, Moore KG, Sartorelli AC. Glycosylation of annexin I and annexin II. Biochem Biophys Res Commun. 1992;188(2):554-8.

19. Dubois T, Oudinet JP, Mira JP, Russo-Marie F. Annexins and protein kinases C. Biochim Biophys Acta. 1996;1313(3):290-4.

20. Mizutani A, Watanabe N, Kitao T, Tokumitsu H, Hidaka H. The long amino-terminal tail domain of annexin XI is necessary for its nuclear localization. Arch Biochem Biophys. 1995;318(1):157-65.

21. Boehm U, Klamp T, Groot M, Howard JC. Cellular responses to interferon-gamma. Annu Rev Immunol. 1997;15:749-95.

22. Li K, Adibzadeh M, Halder T, et al. Tumour-specific MHC-classII-restricted responses after in vitro sensitization to synthetic peptides corresponding to gp100 and Annexin II eluted from melanoma cells. Cancer Immunol Immunother. 1998;47(1):32-8.

23. Kraus M, Romisch J, Bastian B, Paques EP, Hartmann AA. Detection of human anti-annexin autoantibodies by enzyme immunoassays. J Immunoassay. 1992;13(3):411-39.

24. Duncan R, Carpenter B, Main LC, Telfer C, Murray GI. Characterisation and protein expression profiling of annexins in colorectal cancer. Br J Cancer. 2008;98(2):426-33. 